\title{
PEIERLS INSTABILITY AND CHARGE-DENSITY-WAVE TRANSPORT IN FLUORANTHENE AND PERYLENE RADICAL CATION SALTS
}

\author{
W. Brütting ANd W. Riess \\ Physikalisches Institut und Bayreuther Institut für Makromolekülforschung (BIMF) \\ Universität Bayreuth \\ D-95440 Bayreuth, Germany
}

\begin{abstract}
Fluoranthene and perylene radical cation salts are quasi-one-dimensional conductors, which show a Peierls transition to a charge-density-wave ground state. Radical cation salts with different stoichiometries composed of the aromatic donor molecules fluoranthene $\left(\mathrm{Fa}=\mathrm{C}_{16} \mathrm{H}_{10}\right)$ or perylene (Per $=$ $\mathrm{C}_{20} \mathrm{H}_{12}$ ) and the counterion $\mathrm{X}^{-}=\mathrm{PF}_{6}^{-}$were investigated. We find that the temperature dependence of the electrical conductivity and the properties in the charge-density-wa ve ground state strongly depend on the composition of the crystals. When fluoranthene is replaced by perylene, the Peierls transition is shifted from $180 \mathrm{~K}$ to $120 \mathrm{~K}$ and the ground state energy gap decreases from values of $120-180 \mathrm{meV}$ to $60-80 \mathrm{meV}$. In the charge-density-wave ground state the threshold field for nonlinear conductivity varies between 0.1 and $1 \mathrm{~V} / \mathrm{cm}$ for different systems. Our results indicate that disorder, three-dimensional coupling and commensurability effects play an important role for the transport properties of these systems.
\end{abstract}

PACS numbers: 71.45.Lr, 71.30.+h, 72.10.Bg, 72.80.Le

\section{Introduction}

Fluoranthene and perylene radical cation salts belong to the larger class of arene radical cation salts $(\mathrm{Ar})_{n} \mathrm{X}$, where Ar stands for a pure aromatic hydrocarbon and $\mathrm{X}$ is a monovalent anion. Due to their chain-like crystal structure and the partial charge transfer from the donor to the acceptor stack these systems are quasi-one-dimensional (Q1D) conductors, which undergo a Peierls transition to a charge-density-wave (CDW) ground state. The CDW ground state has originally been found in inorganic systems like $\mathrm{NbSe}_{3}, \mathrm{~K}_{0.30} \mathrm{MoO}_{3}$ and $\left(\mathrm{TaSe}_{4}\right)_{2} \mathrm{I}$ [1], but meanwhile its fascinating transport phenomena like nonlinear conductivity, conduction noise in the nonlinear state, metastability and frequency dependent 
conductivity have also been found in organic systems like TTF-TCNQ and some derivatives [2], in the fluoranthene radical cation salts $(\mathrm{Fa})_{2} \mathrm{X}$ [3] and very recently in the perylene system (Per) $)_{2} \mathrm{M}(\mathrm{mnt})_{2}$ [4]. In many of these systems both donor and acceptor stack contribute to the conductivity, which often leads to the occurrence of two or more phase transitions. In contrast to this, in the materials investigated in this work the conductivity originates from the existence of only one stack of organic molecules. The counterions $\mathrm{X}^{-}$are only responsible for the charge transfer and therefore for the partial filling of the conduction band, but the counterion stack does not participate directly in the charge transport.

\section{Experimental}

Single crystals have been obtained by electrocrystallization using standard procedures for this family of compounds. Depending on the crystal growth conditions (solvent and temperature) a number of radical cation salts can be obtained in this way [5-7]. In order to compare the transport properties of such systems we restricted ourselves to systems with almost exactly $2: 1$ stoichiometry and the counterion $\mathrm{X}^{-}=\mathrm{PF}_{6}^{-}$. While from the donor fluoranthene $\left(\mathrm{Fa}=\mathrm{C}_{16} \mathrm{H}_{10}\right)$ only the system $(\mathrm{Fa})_{2} \mathrm{PF}_{6}$ can be grown, from perylene $\left(\mathrm{Per}=\mathrm{C}_{20} \mathrm{H}_{12}\right.$ ) we obtained two radical cation salts $(\mathrm{Per})_{2} \mathrm{PF}_{6} \cdot 0.66 \mathrm{THF}$ and $(\mathrm{Per})_{2}\left(\mathrm{PF}_{6}\right)_{1.1} \cdot 0.8 \mathrm{CH}_{2} \mathrm{Cl}_{2}$, where the anion channel also contains solvent molecules of tetrahydrofurane (THF) and dichloromethane $\left(\mathrm{CH}_{2} \mathrm{Cl}_{2}\right)$.

The transport measurements have been performed on needle-like crystals of typical size $3 \times 0.1 \times 0.1 \mathrm{~mm}^{3}$ using the standard four-probe configuration. Four gold pads were evaporated around the crystal and $25 \mu \mathrm{m}$ diameter gold wires were attached to the pads with gold paint. The electrical resistance was measured with a dc technique using a current source (Keithley 220) and a nanovoltmeter (Keithley 181) or an electrometer (Keithley 617), which allows the measurement of resistances over several orders of magnitude.

\section{Results and discussion}

Figure 1 shows the temperature dependence of the dc conductivity of the three systems between room temperature and about $20 \mathrm{~K}$. On different crystals from the same species we find room temperature conductivities in the range $100-1000(\Omega \mathrm{cm})^{-1}$ for $(\mathrm{Fa})_{2} \mathrm{PF}_{6}$ and $(\mathrm{Per})_{2}\left(\mathrm{PF}_{6}\right)_{1.1} \cdot 0.8 \mathrm{CH}_{2} \mathrm{Cl}_{2}$ and typically $10-100(\Omega \mathrm{cm})^{-1}$ for $(\mathrm{Per})_{2} \mathrm{PF}_{6} \cdot 0.66 \mathrm{THF}$. The fluoranthene system and the perylene system grown from THF solution show a similar temperature dependence. Below room temperature the conductivity at first decreases only slowly with decreasing temperature, but then at the Peierls transition, which occurs at very different temperatures in the two systems (indicated by arrows in the left diagram of Fig. 1), the slope of $\sigma(T)$ suddenly changes and the conductivity begins to decrease rapidly due to the gradual opening of an energy gap. At lower temperatures thermally activated behaviour is observed and from the activation energy the ground state energy gap $2 \Delta(0)$ can be determined (right diagram of Fig. 1 ). For $(\mathrm{Fa})_{2} \mathrm{PF}_{6}$ these values are typically in the range $\Delta(0)=60-90 \mathrm{meV}$, while for $(\mathrm{Per})_{2} \mathrm{PF}_{6} \cdot 0.66 \mathrm{THF}$, due to the lower Peierls transition temperature, values 

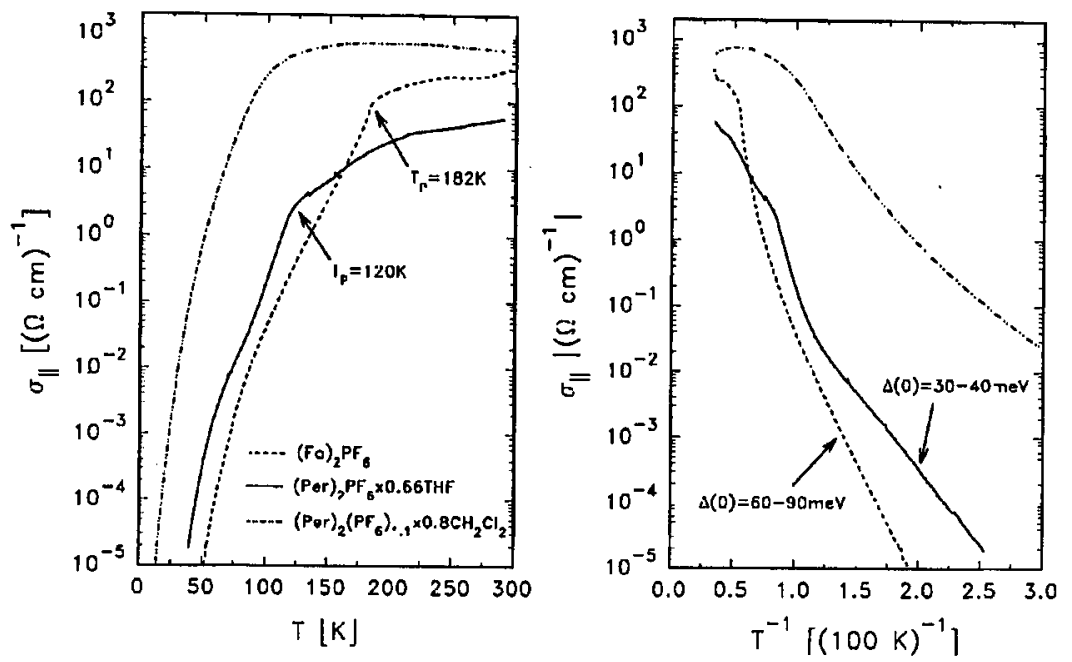

Fig. 1. DC conductivity of $(\mathrm{Fa})_{2} \mathrm{PF}_{6}$ (dashed line), $(\mathrm{Per})_{2} \mathrm{PF}_{6} \cdot 0.66 \mathrm{THF}$ (full line) and $(\mathrm{Per})_{2}\left(\mathrm{PF}_{6}\right)_{1.1} \cdot 0.8 \mathrm{CH}_{2} \mathrm{Cl}_{2}$ (dash-dotted line); in the left diagram the Peierls transition temperatures are indicated by arrows, in the right diagram from the Arrhenius behaviour at lower temperatures the ground state energy gap $2 \Delta(0)$ can be derived.

of $\Delta(0)=30-40 \mathrm{meV}$ are observed. The perylene system from $\mathrm{CH}_{2} \mathrm{Cl}_{2}$ shows a rather different temperature dependence. At first the conductivity increases with decreasing temperature and reaches its maximum between 200 and $150 \mathrm{~K}$. Then there also occurs a change from metallic to insulating behaviour, however, no such sharp phase transition as in the other two systems is observed. Furthermore, the typical Arrhenius behaviour at lower temperatures is not seen (right diagram of Fig. 1).

In (Per) ${ }_{2} \mathrm{PF}_{6} \cdot 0.66 \mathrm{THF}$ there occur two additional phase transitions of first order in the temperature range above the Peierls transition (120-300 K), which can be clearly recognized when the conductivity is plotted linearly versus the ternperature (Fig. 2). At the $220 \mathrm{~K}$ phase transition the slope of $\sigma(T)$ changes and at $155 \mathrm{~K}$ a sudden increase in conductivity is observed. From crystal structure and ESR measurements it is supposed that the phase transition at $220 \mathrm{~K}$ is due to an ordering process in the solvent molecules and the one at $155 \mathrm{~K}$ due to a slight rotation of neighbouring perylene molecules in the conducting stack [8]. The step-like increase in conductivity shows that the systems investigated react very sensitively on a change in the molecular orbital overlap. These phase transitions are also seen in other experiments like differential scanning calorimetry, static magnetic susceptibility and microwave conductivity [8]. Especially the temperature dependence of the microwave conductivity measured at $10.2 \mathrm{GHz}$ shows the same behaviour as the dc conductivity in the temperature range from room temperature to $50 \mathrm{~K}$. This indicates that the observed effects are not due to the influence of contacts (mechanical stress), which can sometimes be a source of artefacts (resistivity jumps) in orgànic conductors. 


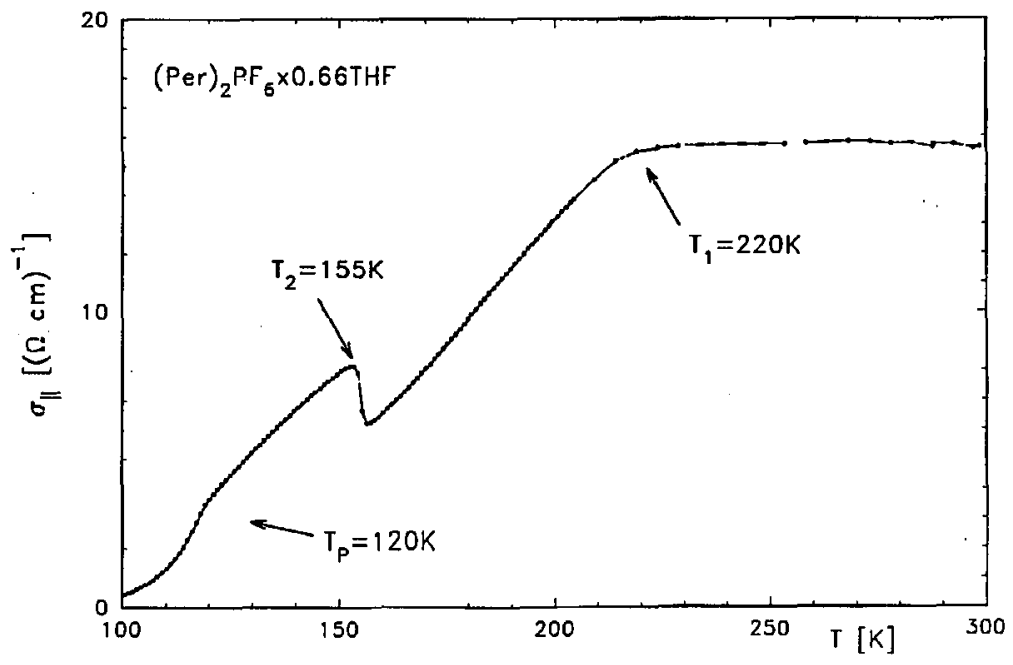

Fig. 2. DC conductivity of $(\mathrm{Per})_{2} \mathrm{PF}_{6} \cdot 0.66 \mathrm{THF}$ in the temperature range above the Peierls transition; the Peierls transition at $T_{\mathrm{P}} \approx 120 \mathrm{~K}$ and the two other structural phase transitions at $T_{1} \approx 220 \mathrm{~K}$ and $T_{2} \approx 155 \mathrm{~K}$ are indicated by arrows.

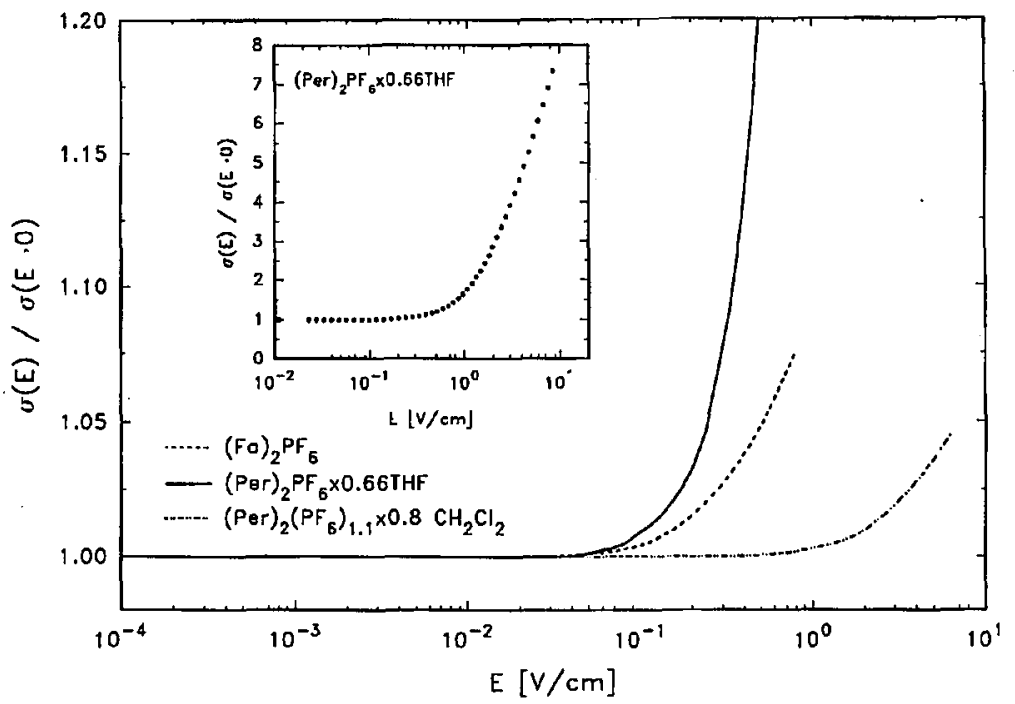

Fig. 3. Nonlinear conductivity of $(\mathrm{Fa})_{2} \mathrm{PF}_{6}$ (dashed line), $(\mathrm{Per})_{2} \mathrm{PF}_{6} \cdot 0.66 \mathrm{THF}$ (full line) and $(\mathrm{Per})_{2}\left(\mathrm{PF}_{6}\right)_{1.1} \cdot 0.8 \mathrm{CH}_{2} \mathrm{Cl}_{2}$ (dash-dotted line) measured at temperatures well below the respective Peierls transition; the insert shows the strong nonlinearity of $(\mathrm{Per})_{2} \mathrm{PF}_{6} \cdot 0.66 \mathrm{THF}$ with a dynamic range of almost one order of magnitude for electric fields of $10 \mathrm{~V} / \mathrm{cm}$. 
One evidence for a CDW ground state is the occurrence of nonlinear conductivity for temperatures well below the Peierls transition. In Fig. 3 the conductivity is plotted as a function of the electric field for the three systems. For low fields the samples show ohmic behaviour, this means field independent conductivity. However for fields exceeding a certain threshold the conductivity increases and nonlinear conductivity is observed due to the contribution of the electrons condensed in the CDW. Due to the high one-dimensionality of these systems (e.g. $\sigma_{\|} / \sigma_{\perp}=10^{3}-10^{4}$ for $\left.(\mathrm{Fa})_{2} \mathrm{PF}_{6}[9]\right)$ and defects no sharp threshold field for nonlinear conductivity occurs. It can be seen that the systems show differences in the onset of the nonlinearity and also in the slope of $\sigma(E)$. The perylene system from $\mathrm{CH}_{2} \mathrm{Cl}_{2}$ shows the highest value of the threshold field and the weakest nonlinearity. Together with the behaviour of the dc conductivity (no sharp Peierls transition) this indicates that disorder plays an important role in this system. The strongest nonlinearity is observed in the perylene system from THF, which shows an increase in conductivity by one order of magnitude for $E \approx 10 \mathrm{~V} / \mathrm{cm}$ (see insert in Fig. 3). This dramatic effect gives evidence that (Per $)_{2} \mathrm{PF}_{6} \cdot 0.66 \mathrm{THF}$ is an incommensurate CDW system - in contrast to $(\mathrm{Fa})_{2} \mathrm{PF}_{6}$, where our nonlinear [3] and frequency dependent experiments [10] together with X-ray investigations [11] indicate the presence of a commensurate CDW ground state.

\section{Conclusion}

Due to their simple molecular and crystal structure fluoranthene and perylene radical cation salts can serve as model systems of Q1D conductors for studying the Peierls instability and the CDW ground state. By variations of the donor molecule or the stoichiometry the transport properties can be influenced significantly. Our investigations show that disorder, three-dimensional coupling and commensurability effects play an important role in the dynamics of these systems. Further investigations are in progress in order to confirm the existence of an incommensurate CDW in the organic radical cation salt $(\mathrm{Per})_{2} \mathrm{PF}_{6} \cdot 0.66 \mathrm{THF}$.

\section{Acknowledgments}

We thank M. Schwoerer and E. Dormann for helpful discussions and H. Dragan and J. Gmeiner for growing the crystals. This work was supported by BASF/BMFT and the Fonds der Chemischen Industrie.

\section{References}

[1] For a review on CDW transport see: Charge-Density-Waves in Solids, Eds. L.P. Gorkov, G. Grüner, North-Holland, Amsterdam 1989.

[2] R.C. Lacoe, H.J. Schulz, D. Jerome, K. Bechgaard, I. Johannsen, Phys. Rev. Lett. 55, 2351 (1985).

[3] W. Rieß, W. Brütting, Phys. Scr. Vol. T 49, 721 (1993).

[4] E.B. Lopes, M.J. Matos, R.T. Henriques, M. Almeida, J. Dumas, Europhys. Lett. 27, 241 (1994). 
[5] V. Enkelmann, J. Phys. (Paris) C9 44, 1147 (1983).

[6] H.J. Keller, D. Nöthe, H. Pritzkow, D. Wehe, M. Werner, P. Koch, D. Schweitzer, Mol. Cryst. Liq. Cryst. 62, 181 (1980).

[7] D. Schweitzer, I. Hennig, K. Bender, H. Endres, H.J. Keller, Mol. Cryst. Liq. Cryst. 120, 213 (1985).

[8] M. Burggraf, H. Dragen, P. Gruner-Bauer, H.W. Helberg, W.F. Kuhs, G. Mattern, D. Mueller, W. Wendl, A. Wolter, E. Dormann, Z. Phys. B, 1994, to be published.

[9] W. Brütting, W. Rieß, M. Schwoerer, Ann. Phys. (Leipzig) 1, 409 (1992).

[10] W. Brütting, G. Witt, A. Rötger, W. Rieß, Proc. ICSM 1994, to be published in Synth. Met., 1995.

[11] V. Ilakovac, S. Ravy, J.P. Pouget, W. Rieß, W. Brütting, M. Schwoerer, J. Phys. IV (Paris) C2 3, 137 (1993). 